record in real-time, placing the most relevant and time critical information at the centre of patient care.

GOSH Link is a web based, clinician facing portal, providing 'external to GOSH' care providers with access to a patient's GOSH digital electronic medical record, regardless of the digital maturity of the external hospital. Using GOSH Link clinicians can view the patient's: encounters, allergies, problems, notes, medications, lab results, radiology reports. Furthermore, using GOSH Link external care providers can upload documents (request to upload documents) to the patient's GOSH record, with further potential to engage actively with the patient's treatment at GOSH.

Without GOSHLink the local hospital team would have to rely on the family or GOSH haematology/Oncology team letting them know of any future appointments/interventions, followed by the initiation of an appropriate management plan. This could have added additional days to the patient's overall care plan through finding out information, or rescheduling the procedure

Being one 'virtual' team- The PTC and POSCU relationship has always been at the very core of facilitating excellent patient care between 2 centres, and good communication is vital for this. GOSHLink has facilitated all of this.

Clinical governance- real time documentation that both the PTC and POSCU can view

Patient experience-families are reassured to know there is electronic documentation of discussion between PTC and POSCU.

Rolling out GOSHLink to POSCU PTC is so much more efficient, saves on time spent chasing appointments by email or telephone. Improves patient safety, and communication. Better patient care. Prevents procedures being cancelled or postponed. GOSHLink supports the response to the COVID19 pandemic between GOSH and local hospitals.

\section{QUALITY IMPROVEMENT INITIATIVE: REDUCING UNNECESSARY STOOL TESTING AND RELATED LABORATORY COSTS}

Malti Nakrani, Wisdom Musabaike, Christine Morris, Nuwanthi Yapa Mahathanthila. Great Ormond Street Hospital

\subsection{6/archdischild-2020-gosh.52}

Background Stool samples sent to the lab without an indication for screening was in the top 5 rejections in the hospital. Approximately 440 stool samples were rejected annually in the Microbiology lab for being sent for testing too frequently. A majority of these rejections were from wards with immunocompromised patients. Requisition/testing unnecessary samples leads to negative patient experience and unnecessary healthcare costs. A stool microscopy and culture costs $£ 21.00$. This is quite significant compared to the number of tests requested.

Our aim was to investigate ward practices in sending stool samples for testing and the causes for high rejection rates of these.

Method Current policies and practices for stool testing in wards and labs were studied, especially the weekly screen for neutropenic patients. Historic and live data were reviewed. Various teams were interviewed to understand the requirements for stool testing. Key groups involved: Infection Prevention and Control (IPC), Laboratory, Microbiologists, Specialty leads and nurses. Ethical clearance was not needed.
Results We found mismatch in protocols held with different departments. We identified discrepancy of policy versus practices and lack of understanding between ward and the lab. We observed inconsistencies between wards.

While many samples were rejected, we also found many were unnecessarily tested.

Discussion Results indicated that a coherent update in departmental policies is required between IPC, Microbiology Laboratory and Nursing teams. Due to significant cultural implications, multiple approaches to educate staff in the updated practice will be required. A special focus is needed to the decision making at the point of requisition. Solutions such as simple flow charts, system updates to aid optimal test requisition on the electronic requesting system and education sessions will be explored in the next part of this study.

Conclusion Differences in practices, guidance and its interpretation led to a significant number of unnecessary testing.

\section{DISCHARGE SUMMARY (SIGHT \& SOUND): ARE WE MEETING THE GOSH TARGET?}

Sevasti Konstantinidou, Miane Min Yan Ng, Bushra Zia, Christopher Jephson. Great Ormond Street Hospital

\subsection{6/archdischild-2020-gosh.53}

Objective In order to facilitate safe management of complex patients following discharge from GOSH, the Trust Timeline Target is to send all discharge letters within 24 hours. This project aims to assess Sight and Sound Directorate's compliance with this standard, identify potential causes of non-compliance and implement sustainable change.

Methods Retrospective data collection was performed via Epic system. The first audit cycle started in September 2019 and the second was completed in August 2020. Due to suboptimal initial results, areas of weakness were identified and changes were implemented. Audit results and educational materials were disseminated within the directorate to emphasise the importance of timely completion and distribution of discharge letters. Directorate managers worked closely with administrative team and a new administrative role was created to facilitate adequate weekend cover.

Results The initial results were worrisome, as none of the departments met the Trust Timeline Target. The percentage of discharge summaries send within 24 hours ranged from $0 \%$ to $88.24 \%$, with an average of $54.4 \%$ across the departments. The majority of letters were completed in a timely manner, but the summaries of the weekend discharges were rarely sent within 24 hours. Following the implementation of changes, the compliance improved significantly, with the proportion ranging from $69.3 \%$ to $100 \%$. Three departments achieved $100 \%$ compliance with the standard and the average percentage was $89.5 \%$.

Discussion This project demonstrates that Sight and Sound Directorate was initially not meeting the Trust Target for discharge letters. This was mainly due to inadequate administrative staff cover over the weekend. After changing that and high-lightening to our teams the importance of sending discharge summaries within 24 hours, the compliance with the GOSH standard drastically improved. We aim to continue monitoring the situation to ensure that the results we achieved are sustainable. 